\title{
Perspective
}

PERSPECTIVE Actualité en histoire de l'art

1 | 2010

Ornement/Ornemental

\section{Flore sacrée : les reliquaires à paperoles, ornements sublimes}

Sacred flora: quilled reliquaries, sublime ornaments

\section{Céleste Olalquiaga}

\section{CpenEdition}

1 Journals

\section{Édition électronique}

URL : http://journals.openedition.org/perspective/1232

DOI : $10.4000 /$ perspective. 1232

ISSN : 2269-7721

Éditeur

Institut national d'histoire de l'art

\section{Édition imprimée}

Date de publication : 30 juin 2010

Pagination : 151-157

ISSN : 1777-7852

\section{Référence électronique}

Céleste Olalquiaga, «Flore sacrée : les reliquaires à paperoles, ornements sublimes », Perspective [En

ligne], 1 | 2010, mis en ligne le 14 août 2013, consulté le 01 octobre 2020. URL : http://

journals.openedition.org/perspective/1232; DOI : https://doi.org/10.4000/perspective.1232 


\section{Flore sacrée : les reliquaires à paperoles, ornements sublimes}

\author{
Céleste Olalquiaga
}

"Lorsque je suis saisi par l'amour de la beauté de la maison de Dieu, la splendeur multicolore des gemmes m'arrache parfois aux soucis extérieurs, et même la diversité des saintes vertus paraît transportée des choses matérielles aux choses immatérielles par une noble méditation, et il me semble que je demeure comme sur quelque plage extérieure à l'orbe terrestre qui ne se trouverait ni dans la lie de la terre ni entièrement dans la pureté du ciel : par le don de Dieu, je suis transporté de l'espace inférieur à cet espace supérieur de manière anagogique " Abbé Suger, 1145-1147'.

Objet de multiples études, notamment pour leur littérature et leurs extases mystiques, les ordres religieux féminins ont été peu analysés sous l'angle de leurs productions matérielles, témoignages pourtant tout aussi passionnants et bien plus tangibles. Parmi cette production se distinguent des objets de dévotion privée d'un type particulier : les reliquaires à papiers roulés, boîtes vitrées figurant une scène religieuse placée au cour d'une végétation luxuriante, dont la première mention apparaît en $1643^{2}$. Qu'il s'agisse de figurines en pâte ou de gravures découpées, des images relevant de l'iconographie chrétienne y sont fixées à la colle animale et entourées de reliques sacrées, elles-mêmes " habillées " par un foisonnement floral fait de papiers roulés, appelés "paperoles ». Encadrés et mis sous verre, ces tableaux en trois dimensions donnent l'impression de boîtes magiques évoquant des bois enchantés (fig. 1).

Comme une grande partie de la création dévotionnelle, la production d'œeuvres à paperoles au sein des ordres féminins tels que les Carmélites, les Ursulines ou la congrégation des Visitandines, a prospéré entre le Concile de Trente (1534-1565), qui légitima et incita même à la production de cette iconographie pieuse, et le Concile Vatican II (1962-1965), lequel sécularisa les rites liturgiques et cultuels au point de les supprimer. Les paperoles furent surtout produits en abondance entre 1720 et 1850 et connurent un véritable essor en Bavière, dans le nord de l'Espagne et dans le sud de la France.

Offerts par les nonnes aux patrons de leurs couvents ou à leurs propres familles, ces objets de culte font partie intégrante du décor domestique, prenant place au sein du foyer, dans une chambre ou encore dans un oratoire privé ; ils ne restent jamais dans les couvents, leurs qualités ornementales ne s'accordant pas avec l'austérité monastique ${ }^{3}$. Réalisés dans des matériaux pauvres, ils sont d'une grande fragilité : la lumière fait fondre les cires et les colles utilisées dans l'élaboration des boîtes et décolore les papiers, les insectes dévorent les unes et les autres, et l'humidité et les champignons achèvent la tâche, s'intégrant presque au décor. Ces objets s'inscrivent ainsi dans une triple précarité cultu(r)elle : découlant d'une filiation pour le moins polémique, celle du culte voué aux reliques, et réalisés par des femmes ne disposant que de matériaux pauvres (selon l'interdiction des ordres contemplatifs féminins d'utiliser des métaux ou des pierres précieuses $)^{4}$, les reliquaires à papiers roulés sont, enfin, péché des péchés, des objets ornementaux de par leur exubérance visuelle. On comprend qu'à la suite de Vatican II, ils aient été massivement jetés à la poubelle.

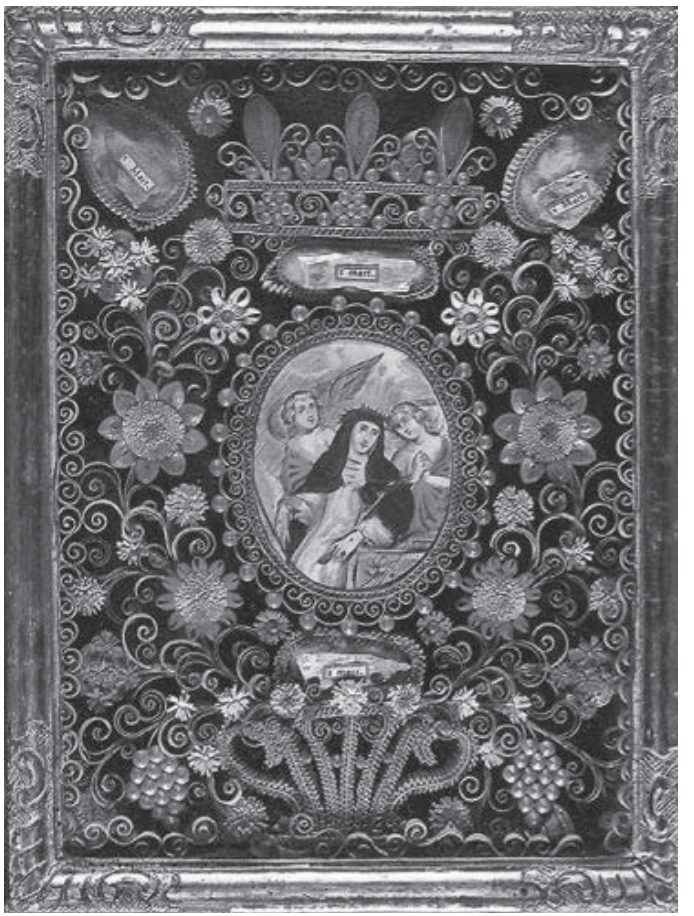

1. Reliquaire à paperoles d'origine flamande (ordre des Carmélites) figurant sainte Catherine de Sienne, XVII ${ }^{\mathrm{e}}$-XVIII ${ }^{\mathrm{e}}$ siècles, gravure sur parchemin rehaussée de gouache, os, papier, carton. 


\section{Boîtes vitrées, mondes enfermés}

Paperoles et reliques tissent des allégories du Paradis, lieu protecteur où l'âme peut résider en toute pureté, à l'image des couvents au sein desquels ces objets sont produits. Les paperoles seraient une version sophistiquée des "petits paradis ", des boîtes vitrées d'une confection rudimentaire souvent centrées sur l'Enfant Jésus ${ }^{5}$. Proches des crèches traditionnelles, ces "paradis "- aussi appelés " jardins ", " grottes ", ou "déserts " selon le style et la composition -, qui ne contiennent ni reliques ni papiers roulés, constituent une variante primitive des reliquaires à paperoles, même si leur côté raboteux les rend souvent davantage oniriques. Troisième déclinaison de ces mondes fermés, les "boîtes de nonnes " en seraient la représentation la plus sévère et minimale : mises en scènes ascétiques, elles montrent la vie des religieuses dans leurs cellules d'une façon tellement dépouillée qu'elles n'ont même pas recours au verre (fig. 2$)^{6}$.

Dès le XIII ${ }^{\mathrm{e}}$ siècle, le célibat féminin s'intensifie et prend la forme métaphorique du jardin clos ou hortus conclusus du Cantique des Cantiques, un paradis en miniature qui représente la vertu parfaite, l'endroit où la moniale peut rejoindre son « Époux céleste ». Lors de sa Résurrection, Jésus se présenta à Marie-Madeleine habillé en jardinier : « Noli me tangere ", ne me touche pas, prévient-il, en pleine transmutation métaphysique de mortel en immortel, du matériel en symbole. Le sacrifice du Christ, qui accepte de racheter les failles de l'humanité,
2. Boîte de nonne montrant une cellule de Carmélite avec

I'inscription «Le plaisir de mourir sans peine veut bien la peine de vivre sans plaisir", XIXe siècle, coll. part, gravures et carton.

3. Icône de Nikita Pavlovets, Le jardin de la Très Pure Mère de Dieu, la vigne enclose, 16701677, Moscou, Galerie Tretiakov. est réitéré par Marie-Madeleine qui veut se rapprocher d'un homme en train de devenir fantasme. Désormais, il lui est interdit de le toucher, geste clé d'une foi à toute épreuve que la moniale reprend dans ses vœux ${ }^{7}$. Imaginaire par définition, l'hortus conclusus concrétise cette pratique cultuelle, recréant

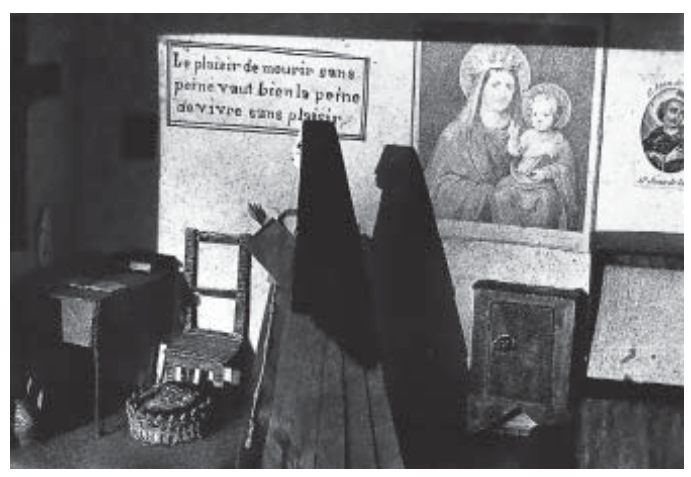

le cloître ou le foyer virginal (fig. 3). Il prend forme dans les Pays-Bas du Sud au XVe siècle, matérialisé en des retables (alors désignés comme tels car placés sur les autels) où sont réunies des reliques au milieu $\mathrm{d}^{\prime}$ une grande floraison ${ }^{8}$.

Le mariage mystique est la version domestiquée, romantique et joyeuse du courant doloriste qui prit son essor au XIII ${ }^{e}$ siècle dans le nord de l'Europe. Fondé sur l'amour du Christ et la contemplation de sa Passion, le dolorisme était centré sur les images de celle-ci et les moments les plus durs de la Via Crucis (la Flagellation et l'Ecce Homo), au point de donner lieu au phénomène de la réception des stigmates. Condamné pour ces excès corporels, le dolorisme prit par la suite un tournant plus doux, sous la forme d'une identification avec les femmes proches du Christ (surtout Marie et Marie-Madeleine) et un échange symbolique de cours entre l'Époux céleste et les religieuses dans le jardin clos.

La tradition dévotionnelle féminine s'est ainsi transférée d'une identification corporelle avec la Passion vers sa sublimation visuelle. Le corps reste central dans cette pratique dévotionnelle, mais il s'agit d'un corps dans sa dimension la plus épurée, écrin du cœur, organe de l'amour divin et terrestre, objet de l' " échange » entre le Christ et la moniale, entre la Vierge et son jardinier. Les religieuses n'essayaient pas, comme jadis, de se rapprocher du Christ en reproduisant son martyr, mais plutôt en ne faisant qu'un avec lui à travers un mariage symbolique dont elles sont à la fois sujet, objet et lieu : épouse, cœur et jardin. Cette triade remplit l'espace $\mathrm{du}$ reliquaire sous la forme d'ornements floraux métaphoriques : les vertus liées à la virginité prennent ainsi la forme de lys, de roses ou de muguet dans des " bouquets spirituels".

Symboles de la pureté, les fleurs représentent aussi l'éphémère de la vie et donc le superficiel,

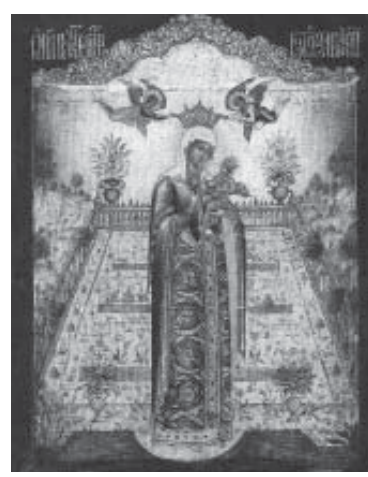

le féminin étant invariablement lié à un de ces sens au moins, selon les cultures et les époques ${ }^{9}$. Dénigrées en tant que motifs décoratifs (de la "fioriture"), les fleurs et, par extension, le féminin demeurent des 
valeurs artistiques secondaires : source, produit ou objet de l'inspiration, elles en sont rarement le sujet. Dans les reliquaires, les fleurs sont faites de "paperoles", des bandes de papiers de différentes épaisseurs et largeurs, colorées et dorées sur tranche et ensuite pliées, plissées, frisottées, roulottées et entortillées. Leur confection minutieuse et répétitive exige un temps long. Cette "prière manuelle " prend la place des instruments de martyr ; le fouet est transformé en guirlande. Si la méthode et les moyens sont nouveaux, le désir et la jouissance restent équivalents. Il ne s'agit plus du processus d'épuration doloriste associé à la Crucifixion mais de son aboutissement dans la délivrance des pêcheuses, désormais vertueuses et prêtes à une union mystique avec leur Époux. À rebours de la chronologie chrétienne, le sacrifice du corps du Christ anticipe celui de la sexualité ou plutôt de l'asexualité paradisiaque, représentée par la Vierge Marie, " rose sans épines " parce qu'indemne du péché originel.

Se déclenche ainsi un florilège spéculaire ${ }^{10}$ où scènes religieuses et fleurs se renvoient les unes aux autres, reflet sémiotique qui produit des tableaux surchargés d'une signification paradoxale : la virginité y est représentée comme une prolifération de fleurs sans fruits, répétition à l'infini d'un geste impossible. Dans ces aléas d'union mystique, le pliage obsessionnel des papiers roulés soutient le poids du sacrifice des religieuses. Seuls ou formant des grappes, des bouquets, des vases ou même des oiseaux, les paperoles enveloppent les reliques, entourent les images, allongent les marges des tableaux et, souvent, sont les éléments constitutifs des autels, arcades ou chapiteaux du décor. Ils proposent un paysage tridimensionnel à perspective multiple, le jeu avec les papiers pliés offrant la possibilité d'entrecroiser et de superposer les éléments. C'est un paroxysme floral qui s'ouvre et se referme en accordéon comme un sourire permanent, offrant une géométrie parfaite mais également irrégulière et débordante, fenêtre ouverte à la fuite des fleurs.

\section{Des ornements transitionnels}

Les reliquaires à paperoles sont des objets transitionnels, non seulement entre catégories d'objets artistiques (objet de culte/objet d'exposition $\left.{ }^{11}\right)$, mais aussi en tant que cristallisation du déplacement libidinal : dans les reliquaires à paperoles, l'élan dévotionnel devient sublimation. $\mathrm{Au}$ cour de ce glissement continu d'un registre à l'autre (cultuel/érotique/artistique) se trouve la libido ${ }^{12}$, matérialisée en couches allégoriques. Dans ce cadre, on pourrait ainsi considérer le décoratif comme une catégorie intermédiaire entre le culte, à l'origine de l'expression artistique en général et de la production animiste et religieuse en particulier, et l'exposition, dans sa condition moderne ou "laïque ", c'est-à-dire dépourvue du sens spirituel en faveur du sens esthétique.

Ces deux formes de pratique culturelle - le culte et l'exposition -, auxquelles un même objet peut appartenir selon l'époque et surtout selon l'usage qu'on lui confère, peuvent se retrouver simultanément dans l'ornatus, ou ornement religieux. Issu du culte, celui-ci ne peut être exposé comme un quelconque objet, soumis au regard distancié que l'on porterait sur une ouvre d'art. En effet sa charge dévotionnelle, animiste ou libidinale (qui tient à sa condition de fétiche ${ }^{13}$ ) dépasse son caractère représentatif et le rapproche immédiatement du spectateur. Cette proximité est encore augmentée par le sens des ornements religieux, qui ne relèvent pas de l'abstraction symbolique, mais d'une sorte de narrativité allégorique apte à produire une atmosphère imaginaire propre aux décors et à la mise en scène ${ }^{14}$.

Parure du corps ou de son extension (à savoir la maison, ecclésiastique ou laïque), l'ornement entre en osmose avec ceux qui le portent ou avec son contexte au point de faire disparaître les différences qui les séparent, objet et sujet devenant une seule et même entité. En tant que parure, l'ornement entraîne un changement d'apparence de la surface dans laquelle il s'intègre. Il est alors intimement lié au toucher, un sens en chute libre depuis l'ère moderne et le primat de la vue. Dans le cas des reliquaires à paperoles, la tactilité leur est presque consubstantielle : de confection manuelle, ils sont également sensibles à cette " caresse oculaire ${ }^{15}$ propre aux ouvres de dévotion. Cette caresse se dessine dans le sens d'un toucher qui relève de l'affectif mais aussi du libidinal, l'objet en question étant instrument et sublimation du désir, d'où son caractère fétichiste.

Les reliquaires à papiers roulés se trouvent donc dans une situation paradoxale : en dépit 


\author{
4. Reliquaire de \\ la Plaie du côté \\ du Christ portant \\ I'inscription \\ "CHARITAS " \\ et provenant \\ du couvent de \\ la Visitation de \\ Dijon, début du \\ XVIIII siècle, os, \\ papier, carton, \\ verre et bois \\ Six médaillons \\ contiennent \\ les reliques.
}

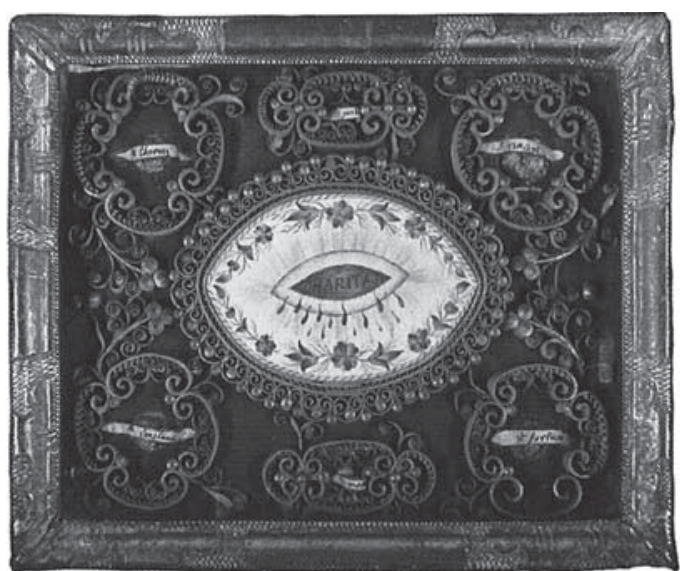

de leur charge émotionnelle (ou précisément à cause d'elle), ils peinent à être légitimés en tant qu'œuvres d'art. En outre, le caractère ornemental de ces objets de dévotion les relègue au statut supposé inférieur d'objet artisanal ou décoratif, seuls la peinture (les icônes) et les trésors (les reliquaires) faisant preuve d'exception dans ce domaine. La parure ostentatoire des paperoles leur donne d'ailleurs un caractère ambivalent, puisqu'elle est conçue tout autant pour susciter que pour dissimuler le désir. Ce rôle rappelle celui des pierres précieuses dont la lumière était censée capter et dévier le mauvais oeil de la jalousie ${ }^{16}$.

Les reliquaires à paperoles illustrent le point de convergence entre attirance et rejet, désir et interdit, une ambivalence qui les marque depuis leur conception jusqu'à leur réception. Conçus avec et à travers le toucher, leur apparence ornementale vise à défendre le contact physique, un rappel du " Noli me tangere " fondamental pour la pratique des moniales. La confection des reliquaires à paperoles par les religieuses manifeste ainsi leur propre élan vital projeté dans la répétition obsessionnelle de cette scène fondatrice. Narrativité, matériaux et gestes créateurs sont autant de signes d'une absence fondamentale, qui permettrait de légitimer, comme par défaut, l'accès de ce type de reliquaire au statut d'œuvre d'art (fig. 4).

\section{L'éclat du divin}

Le rôle des reliques dans ces œuvres est très différent de celui qu'elles jouent dans les reliquaires classiques (châsses en verre, en métal ou simples médaillons contenant des ossements humains). En effet, dans les reliquaires à paperoles, elles ne sont plus l'objet primordial mais un élément parmi d'autres appartenant à une composition al-

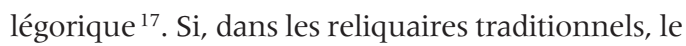
lien avec le Christ est établi à travers les fragments corporels des saints, la liaison entre le terrestre et le divin dans les reliquaires à paperoles est nouée à travers la scène du jardin clos. Certes les reliques y répandent leurs vertus protectrices, mais elles restent toutefois en marge de la narration principale de ces jardins, explicite ou implicite, qu'est le mariage mystique. Cette fonction d'accompagnement à laquelle sont reléguées les reliques est mis en évidence par la place qu'elles occupent dans ces compositions : disposées autour de la scène centrale, elles ont toujours un lien avec elle.

Les reliques et les fleurs à paperoles entament un dialogue particulier dans ces reliquaires. Les premières étant souvent difficiles à discerner au milieu des secondes, elles ne peuvent généralement être repérées que par les étiquettes manuscrites affichant les noms de leurs saints correspondants et passent ainsi à l'arrière-plan. Les reliques deviennent alors ornementales, tandis que les fleurs de papier qui les entourent pour mieux les mettre en valeur se transforment en matière sacrée par le simple fait d'entrer en relation avec ces vestiges auréatiques ${ }^{18}$.

Manipulées et investies de significations jusqu'à en donner le vertige - symboles des vertus, allégories du Cœur mystique, "prières manuelles ", reliquaires - les fleurs à paperoles renvoient l'ornatus à son statut sacré original, dont le fond et la forme sont inséparables, empêchant une hiérarchie entre images, reliques et fleurs. En produisant une charge dévotionnelle par le biais de leur narrativité et leurs matériaux, mais aussi par le geste silencieux et répétitif de leur processus de fabrication, les paperoles deviennent elles-mêmes des objets de piété, une véritable flore sacrée : non pas un moyen pour atteindre un but, mais un but en soi ; non pas une représentation, mais une présence.

Tout en réunissant les modes de représentation des reliques et des icônes, les reliquaires à paperoles conservent leur singularité. Les reliques, authentiques ou fausses (peu importe, puisqu'il s'agit d'actes de foi), proposent un lien spirituel par la matière, établissant une chaîne métonymique où le rapprochement avec le Christ a lieu à travers 
des fragments chargés de sens sacré. Posséder ou s'approcher de ces reliques, même s'il n'y a pas de contact direct, est une manière de participer à cet enchaînement divin. Les icônes, au contraire, privilégient le lien spirituel via l'intellect. Le panneau peint est alors le support d'une image codifiée qui conduit à l'expérience transcendantale ; il n’y a pas de rapprochement physique, même si, parfois, les icônes sont touchées ou explicitées par un récit écrit ${ }^{19}$. Les reliques impliquent en effet une tangibilité, un toucher, même s'il n'est qu'imaginaire et résiduel, tandis que le parcours spirituel des icônes se déroule strictement à travers le regard. L'expérience visuelle et spirituelle de l'icône diffère donc de celle des reliques, et l'on comprend que l'essor des icônes au VIII ${ }^{\mathrm{e}}$ siècle ait été une véritable révolution culturelle accompagnée par des lois, des bannissements et des guerres.

À l'inverse, reliquaires, icônes et reliquaires à paperoles partagent un élément clé, à savoir le reflet ou rayonnement, produit par l'incrustation de bijoux dans la châsse en métal des reliquaires et par la peinture dorée des icônes, souvent ellesmêmes revêtus de métal. Le rayonnement lumineux de ces objets de culte en renforce la valeur sacrée, de même qu'un halo sacré auréole les saints et les vierges dans les icônes. Les matériaux brillants tels que le cristal, le verre, l'or, l'argent et les pierres précieuses, rendent les objets littéralement resplendissants - des rayonnements qui sont aussi de métaphores du corps divin ${ }^{20}$. Cette même lueur sacralisante des métaux et des bijoux rehausse la présentation des reliques dans des châsses anthropomorphiques dont la forme, reproduisant celle des membres "mutilés " (bras, torse, tête - autant de substituts qui deviendront des ex-voto réduits au minimum), rappelle l'appartenance organique et le caractère résiduel du fragment, ainsi que le souvenir du martyr (fig. 5).

Cet éclat divin est à double tranchant, puisque la lumière est capable d'aveugler, protégeant les trésors des mains et des regards trop avides. En atteste l'ample utilisation du cristal et du verre dans les reliquaires : matières translucides et réfléchissantes, elles permettent à la fois de regarder les reliques et de les maintenir à distance, tout en accroissant leur valeur. Cette double fonction est réitérée dans les revêtements métalliques des icônes. Telles des armures, ces blindages ne laissent apparaître que les visages et les mains des saints : c'est surtout à travers le regard et le toucher que ceux-ci agissent, comme en témoignent les rayons dorés qui émanent parfois de leurs yeux et de leurs paumes.

Dans leur mise en scène iconographique des reliques, les reliquaires à paperoles mélangent ces traditions dévotionnelles devenues presque antinomiques, même si elles étaient relativement proches de l'iconographie des "icônes reliquaires ${ }^{21}$. Les reliquaires à paperoles recréent les effets auréatiques, à la différence près que leur présentation est en trompe-l'œil. Réalisées obligatoirement à partir de matières pauvres, les paperoles reproduisent les effets des reliquaires et des icônes par des moyens détournés et astucieux. Ainsi, le relief et la tridimensionnalité sont évoqués au moyen de la juxtaposition et de la saturation. Les couches concentriques des pétales en paperoles produisent une floraison débridée qui fonctionne comme un élément d'encadrement, aussi bien à l'intérieur de la composition qu'autour d'elle, entourant les scènes telles des maries-louises ou des passe-partout floraux. En outre, la lueur de métal doré ou argenté des tranches des paperoles rappelle l'éclat des châsses et des pierres précieuses au point que les paperoles donnent l'impression d'être toutes entières faites d'or ou d'argent.

Ornements qui imitent l'ornement, faux décor pour une vraie dévotion, les reliquaires à paperoles et leur surenchère florale s'inscrivent dans le baroque considéré comme un style et non seulement comme l'art d'une époque. Figuratifs, éclectiques et débordants, ces objets franchissent les limites de la représentation en mêlant matériaux et traditions pour donner lieu à des états de

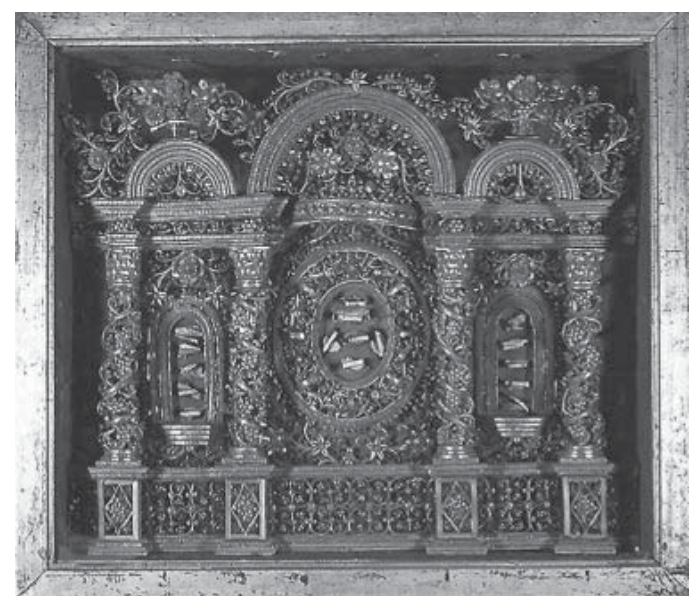

5. Retable avec reliques et paperoles, ordre des Carmélites, XVII - -XVIIII siècles, coll. part. os, papier, ruban rouge, carton. 
rêverie et de fusion, tant pour celles qui les produisent que pour celles et ceux qui les regardent. Flore sacrée qui cache à peine sa vraie nature libidinale et la restitue indirectement dans une fantaisie inouïe, les reliquaires à paperoles offrent une expérience unique de l'excès, tant matériel et visuel que dévotionnel. À ce titre, ils remettent aussi en cause le statut secondaire de l'ornemental ou du décoratif, montrant bien que le sujet et sa mise en scène sont inséparables et renforçant ainsi leur valeur en tant que métaphore d'un élan religieux et esthétique. Images religieuses, reliques et fleurs tournoient comme un manège $\mathrm{du}$ paradis, nous renvoyant en permanence à une scène primaire et interdite : "Noli me tangere ", ou le désir se repliant pour toujours sur lui-même, une plaie qui ne se referme pas.

1. L'abbé Suger, Euvres complètes de Suger, recueillies, annotées et publiées d'après les manuscrits pour la Société de l'histoire de France, Albert Lecoy de La Marché éd., Paris, 1867, p. 198.

2. Nicole Courtine trouve la première référence aux reliquaires à paperoles dans une lettre datée 1643, "Au cour de la piété baroque, les reliquaires domestiques à papiers roulés", dans Reliquaires à papiers roulés des XVII", XVIII", $X I X^{e}$ siècles, (colloque, Chalon-sur-Saône, 2004, et cat. expo., Paris, Bibliothèque Forney, 2005), Chalon-sur-Saône, 2005. p. 13-24. Le livre précurseur dans le genre est celui de JeanFrançois Lefort, Les Paperoles des Carmélites. Travaux de couvents en Provence au XVIII siècle, Paris, 1985. Sur la production conventuelle artisanale plus générale, voir Henri Brémond, Histoire du sentiment religieux en France depuis la fin des guerres de religion jusqu'à nos jours, II, L'invasion mystique (1590-1620), Paris, (1923) 1967 ; Marius Barbeau, Les Saintes artisanes, 2 vol., numéros spéciaux des Cahiers d'art ARCA, 2-3, 19441956 ; Victor-Lucien Tapié éd., Retables baroques de Bretagne et spiritualité $d u$ XVII siècle, Paris, 1972 ; Michèle Ménard, Une histoire des mentalités religieuses aux XVII et XVIII siècles : mille retables de l'ancien diocèse du Mans, Paris, 1980 ; Geneviève Reynes, Couvents de femmes : la vie des religieuses contemplatives dans la France des XVII et XVIII siècles, Paris, 1987 ; Au-delà du visible, reliquaires et travaux de couvents, Yvonne Lehnherr et al éd., (cat. expo., Fribourg, Musée d'art et d'histoire, 20032004), Fribourg, 2003 (édition bilingue allemand/français).

3. Annik Pardailhé-Galabrun, La naissance de l'intime : 3000 foyers parisiens, XVII $-X V I I I^{e}$ siècles, Paris, 1988.

4. L'injonction d'utiliser des matériaux pauvres vient notamment de Sainte Thérèse d'Avila ; voir Bernard Berthod, "Travaux de couvent, témoins de la spiritualité tridentine ", dans Reliquaires à papiers roulés..., 2005, cité n. 2, p. 25-32.

5. Grottes, déserts, jardins, paradis, boittes vitrées, mondes enfermés, Thierry Pinette, Laure Monnier éd., (cat. expo., Chalon-
sur-Saône, Chapelle du Carmel, 2007), Chalon-sur-Saône, 2007. Cet ouvrage, ainsi que Reliquaires à papiers roulés..., cité n. 2, a été publié par l'association Trésors de Ferveur, créée en 1997, dont le but est de collecter, étudier, restaurer et exposer les objets de piété à usage domestique, réalisés par des religieuses et des artisans du XVII ${ }^{\mathrm{e}}$ siècle à la Révolution, puis de façon moins spectaculaire au XIX ${ }^{\mathrm{e}}$ siècle. Consulter également Christine Hagen, "Les 'petits paradis' ", dans Au-delà du visible..., 2003, cité n. 2, p. 67-74.

6. Voir l'excellente analyse de Jeanne Laporte-Andlalier " Emboîtement ", dans Au-delà du visible..., 2003, cité n. 2, p. 55-66.

7. Je me permets d'associer l'image du Christ en Résurrection à l'image des Cantiques en raison de leurs éléments communs : le toucher, la femme et le jardin. Les deux derniers ne font qu'un dans la tradition du jardin clos. Pour une analyse approfondie de ce rapprochement, voir Paul Vandenbroeck, "Tu m'effleures, moi qui suis intouchable ", dans Le jardin clos de l'âme : l'imaginaire des religieuses dans les Pays-Bas du Sud, depuis le XIIr siècle, Paul Vanderbroeck éd., (cat. expo., Bruxelles, Palais des Beaux-Arts, 1994), Bruxelles, 1994, p. 13-152.

8. Berthod, 2005, cité n. 4, p. 26

9. Voir Elisabeth Hardouin-Fugier, Etienne Grafe, Les peintres de fleurs en France. De Redouté à Redon, Paris, 1992. Pour la symbolique spécifique de chaque fleur, voir Lucia Impelluso, La nature et ses symboles, Paris, 2004, notamment p. 12-15 et 73-131. [éd. orig. : La natura e $i$ suoi simboli: piante, fiori e animali, Milan, 2003].

10. Les "florilèges", recueils d'images tirées d'herbiers - d'où leur nom -, se répandent dans l'Europe du $\mathrm{XVII}^{\mathrm{e}}$ siècle où ils deviennent aussi des formes littéraires ; voir Pedro de Ribadeneyra, Les Fleurs des vies des Saints des festes de toute l'année suivant l'usage du Calendrier romain réformé, Rouen, 1645-1646 [éd. orig : Flos Sanctorum o libro de las vidas de los santos, Madrid, 1599-1601].

11. Au sujet de la distinction objet de culte/objet d'exposition, voir Walter Benjamin, L'auvre d'art à l'époque de sa reproductibilité technique, Paris, 2000, p. 269-316 [éd. orig. : Das Kunstwerk im Zeitalter seiner technischen Reproduziebarkeit, Francfort, 1939].

12. La libido, du latin désir, désigne la manifestation de la pulsion sexuelle dans la vie psychique et ses différents déplacements et sublimations ; voir Jean Laplanche, JeanBertrand Pontalis, Vocabulaire de la psychanalyse, Paris, 2002, p. 295-297. La sublimation sexuelle et/ou le rôle des femmes dans la production artistique religieuse sont analysés dans les essais développés dans Le jardin clos de l'âme..., 1994, cité n. 7 : celui de Paul Vandenbroeck (cité n. 7), de Julia Kristeva ( Le Bonheur des béguines », p. 167-177), et de Birgit Pelzer ("Reliquats », p. 179-203).

13. Les paperoles sont des fétiches dans les deux acceptions du terme : objet animé, doté d'une " âme " (en anthropologie), et objet de substitution et surinvestissement sexuel (en psychanalyse) ; voir Paul-Laurent Assoun, Le fétichisme, Paris, 1994.

14. Raymond Court et al., L'effet trompe-l'ail dans l'art et la psychanalyse, Paris, 1988.

15. Haptisch : La caresse de l'ail, (cat. expo., Les Sables d'Olonne, Musée de l'Abbaye Sainte-Croix, 1993), (Cahiers de l'Abbaye Sainte-Croix, 75), Les Sables d'Olonne, 1993. 
16. De manière similaire, l'ornement naturel que l'on observe chez les animaux est dans certains cas un leurre, une simulation visant à repousser l'ennemi ; voir Roger Caillois, Méduse et Cie., Paris, 1960.

17. Les reliquaires à paperoles partagent avec les reliques sacrées un destin culturel commun. Ces dernières, glorifiées au point de devenir les légitimatrices officielles des autels chrétiens à l'époque de Charlemagne (selon le Concile de Nicée en 787, les autels devaient contenir des reliques pour être sacrés), furent ensuite reniées par Calvin, puis autorisées à nouveau par le Concile de Trente. Voir Jean-Claude Schmitt, Le corps des images : essais sur la culture visuelle au Moyen Âge, Paris, 2002, p. 273-294. De la même manière, d'objet de culte, les reliquaires à paperoles passèrent au statut d'objet déchu avant d'être collectionnés en tant que témoignages d'une époque révolue, pour des raisons qui ont peu à voir avec leur caractère dévotionnel ou talismanique. Lefort place les reliquaires à paperoles dans le contexte moderne des cabinets de curiosités (Lefort, 1985, cité n. 2, p. 71-72).

18. Sur la contagion métonymique des éléments sacrés, voir Adalgisa Lugli, Naturalia et Mirabilia : les cabinets de curiosités d'Europe, Paris, 1998 [éd. orig. : Naturalia et Mirabilia: il collezionismo enciclopedico nelle Wunderkammern d'Europa, Milan, 1983]. Voir aussi Céleste Olalquiaga, "Regardez mais ne touchez pas ! Le rôle de la tactilité à l'ère de la primauté du visuel ", dans Trevor Gould, Posing for the Public: The World as Exhibition/Poser pour le Public, le Monde comme Exposition, Sandra Grant Marchand éd., (cat. expo., Québec, Musée d'Art Contemporain de Montréal/Hamilton, Art Gallery of Hamilton, 1998-2002), Montréal/Hamilton, 2002, p. 159168 (édition bilingue anglais/français), article disponible sur www.celesteolalquiaga.com/regardez.htm.

19. Je pense aux icônes de la tradition votive populaire, par exemple mexicaine, plus proches dans leur figuration narrative des tableaux discutés ici que les icônes classiques de la tradition byzantine. Pour ces derniers, voir MarieJosé Mondzain, Image, icône, économie : les sources byzantines de l'imaginaire contemporain, Paris, 1996.

20. Je propose le verre comme métaphore de l'aura : Olalquiaga, 2002, cité n. 18, et Royaume de l'artifice : l'émergence du kitsch au XIX siècle, Lyon, 2008 [éd. orig. : The Artificial Kingdom: On the Kitsch Experience, New York, 1998]. Schmitt privilégie l'interprétation selon laquelle «les matières précieuses sont comme la chair des reliques qu'elles dissimulent aux regards " (Schmitt, 2002, cité n. 17, p. 286-287). Pour les rayonnements divins, voir Erwin Panofsky, "L'Art nouveau et la métaphysique de la lumière ", dans Architecture gothique et pensée scolastique, Pierre Bourdieu éd., Paris, 1974, p. 33-47 [éd. orig. : Gothic Architecture and Scholasticism, Latrobe (PA), 1951].

21. On trouve de petites "icônes reliquaires" dès le XII ${ }^{\mathrm{e}}$ siècle, probablement une version condensée des icônes et reliquaires pour les voyageurs ; pourtant, elles ont peu à voir avec les reliquaires à paperoles : de la taille de la main, ces objets superposent un fragment de relique à l'icône, ne laissant aucune place au décor.

Céleste Olalquiaga, historienne celeste@celesteolalquiaga.com

\section{Situations du décoratif en France au tournant du XIX ${ }^{\mathrm{e}}$ siècle : norme, unité et suggestion}

\section{Rossella Froissart Pezone}

À l'occasion de la mort de Paul Gauguin, Maurice Denis revenait sur le moment, au début des années 1890, où celui-ci avait libéré le petit cercle des Nabis "de toutes les entraves" en leur montrant la manière $d^{\prime}$ " affirmer jusqu'à la déformation la courbure d'une belle épaule, [d']outrer la blancheur nacrée d'une carnation, [de] raidir la symétrie d'une ramure que n'agite

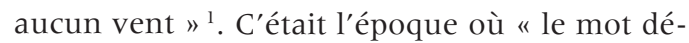
coratif n'était pas encore devenu le 'tarte à la crème' des discussions entre artistes, et même entre gens du monde $"{ }^{2}$. Gauguin avait alors déblayé le terrain du décoratif fin de siècle : au moment où "l'art nouveau et son snobisme n'existait pas encore ", il s'agissait, pour ce Poussin dépourvu de culture classique, de concilier synthèse et style en puisant dans l'archaïsme des calvaires bretons et des idoles maories comme dans le coloriage brutal des images d'Épinal ${ }^{3}$.

Conjonction extraordinaire donc, celle du tournant des années 1880, qui voit le mot décoratif cesser d'être un adjectif pour se nominaliser, en se chargeant, à entendre Denis, des valeurs esthétiques les plus positives qui soient, tant du côté de la grande tradition incarnée par Poussin - les idées de style, de simplicité et de clarté - que de celui de l'avant-garde primitiviste. On peut s'accorder sur le fait que, à ce moment privilégié, la catégorie du décoratif - englobant celle plus restreinte de l'ornement - s'installe en France dans une configuration nouvelle. Grâce à un processus se déroulant, selon Danièle Cohn, sur le temps long qui va de Kant à Worringer, elle devient « le symptôme à partir duquel diagnostiquer la transformation radicale des doctrines classiques de l'art " et finit par se placer au " centre d'un réseau où règnent l'imagination, la liberté, la forme ${ }^{4}$. 